\title{
Proporção de Linfonodos Metastáticos como Variável Independente de Prognóstico no Câncer Colorretal
}

\section{Metastatic Lymph Node Ratio as an Independent Prognostic Variable in Colorectal Cancer}

DENISE GONÇALVES PRIOLLI' ${ }^{1}$ IZILDA APARECIDA CARDINALLI'² CAMILA HELAEHIL ALFREDO; ANA PAULA PIMENTEL SPADARI ${ }^{3}$; FELIPE RODRIGUES MÁXIMO ${ }^{3}$; NELSON FONTANA MARGARIDO ${ }^{4}$; CARLOS AUGUSTO REAL MARTINEZ ${ }^{5}$

${ }^{1}$ Professor Adjunto Doutor do Curso de Medicina da Universidade São Francisco, Bragança Paulista, São Paulo, Brasil;" Professor Adjunto Doutor, Chefe da Disciplina de Patologia do Curso de Medicina da Universidade São Francisco, Bragança Paulista, São Paulo, Brasil; ${ }^{3}$ Acadêmicos do Curso de Medicina da Universidade São Francisco, Bragança Paulista, São Paulo, Brasil; ${ }^{4}$ Professor Livre-Docente do Departamento de Cirurgia da Universidade de São Paulo, São Paulo, Brasil; ${ }^{5}$ Professor Adjunto Doutor do Curso de Pós-Graduação em Ciências da Saúde da Universidade São Francisco. Chefe do Serviço de Cirurgia Geral do Hospital Universitário São Francisco, Bragança Paulista, São Paulo, Brasil.

PRIOLLI DG; CARDINALLI IA; ALFREDO CH; SPADARI APP; MÁXIMO FR; MARGARIDO NF; MARTINEZ CAR. Proporção de Linfonodos Metastáticos como Variável Independente de Prognóstico no Câncer Colorretal. Rev bras Coloproct, 2008;28(4): 431-442.

RESUMO: No câncer colorretal, o comprometimento linfonodal é um dos fatores prognósticos mais importantes. Objetivo: Determinar o valor prognóstico independentemente da relação entre linfonodos comprometidos e examinados em doentes com câncer colorretal. Método: Foram estudados 113 doentes (62 mulheres) portadores de câncer do cólon e reto superior. Quinze pertenciam ao estádio I da classificação TNM, 44 ao estádio II, 42 ao estádio III e 24 ao estádio IV. O índice de linfonodos comprometidos foi determinado pela relação entre número total de linfonodos comprometidos e examinados. Os doentes foram divididos segundo a proporção de linfonodos comprometidos em três grupos: ILC-0: sem comprometimento linfonodal; ILC-1: com comprometimento de até $20 \%$ dos linfonodos examinados; e ILC-2: com comprometimento neoplásico em $21 \%$ ou mais dos linfonodos examinados. As relações entre proporção de linfonodos comprometidos, número de linfonodos ressecados e número de linfonodos comprometidos foram determinadas. Excluíram-se doentes menores de 18 anos, submetidos à neoadjuvância e aqueles onde foram ressecados menos de 12 linfonodos. As variáveis anátomo-clínicas foram analisadas por estatística descritiva. A correlação entre as variáveis foi avaliada pelo teste de Spearman. A sobrevida foi determinada pelos testes de Kaplan-Meier, Log-rank e a análise multivariada pelo modelo de Cox estabelecendo nível de significância de 5\%. Resultados: Houve diferença significante na sobrevida de cinco anos em doentes classificados nos diferentes grupos de ILC $(p=0,009)$. Houve relação entre o número total de linfonodos comprometidos e o ILC ( $(\mathrm{p}=\mathbf{0 , 0 0 0 0 1}$, com $\mathrm{rs}=\mathbf{0 , 9 7 7})$. Doentes do grupo ILC-0 apresentaram sobrevida global em cinco anos maior que $80 \%$, enquanto os pertencentes aos grupos ILC-1 e ILC-2 a sobrevida global foi menor que $60 \%$ e 40\%, respectivamente. $O$ comprometimento linfonodal isolado apresentava-se relacionado à sobrevida em cinco anos $(\mathrm{p}=0,03)$. A análise multivariada demonstrou que o ILC é fator prognóstico independente $(\mathbf{p}=\mathbf{0 , 0 0 9})$. Conclusão: Os resultados do presente estudo permitem concluir que o ILC pode ser considerado uma variável independente preditora de sobrevida em doentes com câncer colorretal.

Descritores: Linfonodos; Excisão de Linfonodo; Prognóstico; Neoplasias colorretais; Análise de sobrevida; Taxa de Sobrevida.

Trabalho realizado pelo Programa de Pós-Graduação em Ciências da Saúde da Universidade São Francisco, Bragança Paulista, São Paulo, São Paulo, Brasil.

Trabalho contemplado com o PRÊMIO AB durante o $57^{\circ}$ Congresso Brasileiro de Coloproctologia realizado em Gramado (RS) em setembro de 2008. 


\section{INTRODUÇÃO}

O câncer colorretal (CCR) atualmente é o segundo câncer mais prevalente em todo o mundo. Estima-se que nos EUA sejam diagnosticados 153.760 casos novos por ano, e que em 2007 ocorreram 52.180 óbitos relacionados à doença. ${ }^{(1)}$ Nos últimos anos variáveis clínicas, histopatológicas, moleculares e genéticas têm sido relacionadas à sobrevida e a sobrevida livre de doença em doentes com CCR. ${ }^{(2-14)}$ Assim, o diagnóstico precoce, ${ }^{(2)}$ a idade, ${ }^{(3)}$ a localização da neoplasia, ${ }^{(4)}$ a graduação histológica, ${ }^{(5)}$ o grau de penetração na parede intestinal, ${ }^{(6)}$ o comprometimento linfonodal, ${ }^{(7,8)}$ a produção de muco pela neoplasia, ${ }^{(9)}$ os níveis pré-operatórios do antígeno carcinoembrionário (CEA), ${ }^{(10,11)}$ a invasão venosa, ${ }^{(12)}$ neural, ${ }^{(13)}$ e o perfil da expressão de vários genes ${ }^{(14)}$ são as variáveis mais estudadas, como fatores relacionados ao prognóstico da doença. Apesar da importância de todas essas variáveis, a invasão parietal, o comprometimento linfonodal e a presença de metástases, continuam representando a principal base de predição da sobrevida e critério para instituição da terapia adjuvante. ${ }^{(7)}$ Embora estudos recentes venham dando ênfase ao comprometimento linfonodal como variável determinante relacionada ao prognóstico de enfermos com CCR, questiona-se se a linfadenectomia poderia realmente produzir efeito benéfico na sobrevida dos doentes ou simplesmente seria procedimento que traria apenas melhor acurácia no estadiamento neoplasia. ${ }^{(15,16)}$

$\mathrm{O}$ efeito adverso do comprometimento dos linfonodos ( $\mathrm{LN})$ na sobrevida de doentes com CCR tem sido uma das justificativas para a indicação de terapia adjuvante em enfermos com doença linfonodal. A menor sobrevida desses pacientes fez com que a indicação de terapias adjuvantes fosse associada ao tratamento cirúrgico, como forma de melhorar estes índices, independentemente do número de LN comprometidos. Dessa forma, doentes com apenas um LN comprometido são submetidos ao mesmo protocolo de tratamento adjuvante dos doentes com envolvimento linfonodal mais extenso.

Estudos em portadores de CCR têm reportado que tanto a sobrevida livre de doença quanto a sobrevida global encontram-se relacionadas ao maior número de LN ressecados. ${ }^{(17)}$ Contudo, o número de LN identificados no espécime cirúrgico encontra-se diretamente relacionado à experiência do cirurgião durante a ressecção da neoplasia, da técnica histopatológica empregada para a sua recuperação, bem como da experiência do patologista em identificá-los. ${ }^{(18)}$ Assim sendo, espécimes cirúrgicos com pequeno número de LN ressecados ou identificados trazem insegurança na determinação fidedigna do comprometimento neoplásico e, conseqüentemente, na indicação do tratamento adjuvante. Com o intuito de diminuir o impacto da ressecção linfonodal tímida, vem sendo estudada a importância da relação existente entre número de LN ressecados e comprometidos pelo CCR. Estudos realizados em doentes com tumores primários do estômago, ${ }^{(19,20)}$ bexiga, ${ }^{(21)}$ mama, ${ }^{(22)}$ pâncreas ${ }^{(23)}$ mostraram relação entre a proporção de LN comprometidos, sobrevida livre de doença e a sobrevida global. Da mesma forma, demonstrou-se que no carcinoma gástrico, a proporção dos LN comprometidos em relação aos examinados, índice de linfonodos comprometidos (ILC), apresenta maior poder prognóstico quando comparada a simples determinação do número de LN comprometidos, adotada pelos diversos sistemas de estadiamento. ${ }^{(24,25,26,27)}$ Diante destas evidências, o emprego do ILC vem sendo proposto com o objetivo de aprimorar e reduzir a margem preditiva de erro em relação à sobrevida e intervalo livre de doença, sobretudo em doentes onde o número de $\mathrm{LN}$ ressecados ou examinados ficou aquém do limite mínimo preconizado. ${ }^{(24,25)}$

Poucos estudos avaliaram o ILC em relação à sobrevida em doentes com CCR e, apenas em dois deles, a avaliação do ILC foi independente do estádio. ${ }^{(24,28)}$ A validade do emprego do ILC como variável relacionada a sobrevida do CCR ainda é controversa. Alguns estudos não mostraram relação ente o ILC e a sobrevida em portadores de $\mathrm{CCR}^{(28)}$, enquanto outros consideram o ILC mais importante do que a simples presença do comprometimento linfonodal. (20,23,24,29) $^{2}$ Desta forma, a importância do ILC como fator prognóstico independente do estadiamento no câncer colorretal ainda merece ser melhor avaliada.

O objetivo do presente estudo foi examinar e determinar o impacto do ILC no prognóstico de doentes com CCR.

\section{MÉTODO}

A realização do estudo obedeceu todas as etapas previstas pelo Comitê de Ética em Pesquisa da Universidade São Francisco e as exigências do Con- 
selho de Ética em Pesquisa da Comissão Nacional de Ética em Pesquisa (CONEP) do Ministério da Saúde (Resolução CNS196/96).

Trata-se de estudo retrospectivo, realizado por consulta à base de dados do Grupo de Coloproctologia do Hospital Universitário São Francisco. Foram considerados elegíveis para o estudo todos os enfermos com diagnóstico de adenocarcinoma do cólon e reto alto (localizados acima da reflexão peritonial), classificados como pertencentes a quaisquer estádios da classificação TNM onde foi realizada a ressecção completa do tumor primário. Foram excluídos os menores de 18 anos e aqueles que apresentavam menos de 12 LN no espécime cirúrgico ressecado. ${ }^{(30)}$ Também se excluíram os portadores de tumor de reto médio e baixo por terem sido submetidos a protocolo de quimioradioterapia neoadjuvante. Após a seleção, de um total de 324 casos foram incluídos no estudo os registros de 113 doentes.

Da base de dados, foram anotadas as seguintes variáveis: idade (maior ou menor de 70 anos), gênero (masculino ou feminino), raça (branca, negra e amarela), grau histológico da neoplasia (bem, moderadamente e pouco diferenciada), tipo da neoplasia (produtor e não produtor de muco), invasão angiolinfática (presente ou ausente), profundidade de invasão da parede cólica $\left(\mathrm{T}_{1}\right.$ a $\mathrm{T}_{4}$ ) estadiamento patológico TNM (I a IV), localização do tumor (cólon proximal, cólon distal e reto alto), número de $\mathrm{LN}$ ressecados (média e mediana), número de $\mathrm{LN}$ comprometidos $\left(\mathrm{N}_{0}, \mathrm{~N}_{1}\right.$ e $\left.\mathrm{N}_{2}\right)$, tempo de seguimento após a cirurgia (em meses), data do óbito, e sobrevida global.

As lâminas selecionadas, sempre coradas pela técnica de hematoxilina-eosina (HE), foram levantadas no Departamento de Patologia e revistas por especialista experiente em neoplasias do trato digestivo para confirmação do diagnóstico anatomopatológico e revisão das variáveis histopatológicas consideradas. A invasão da parede foi avaliada de acordo com a profundidade do comprometimento mucoso, muscular, da serosa ou do tecido adiposo adjacente. A verificação de comprometimento neoplásico dos $\mathrm{LN}$ ressecados foi avaliada pela técnica da HE não se utilizando qualquer técnica imunoistoquímica para pesquisa de micrometástases.

O cálculo do ILC foi determinado pela razão entre número total de LN comprometidos e examinados (ressecados), subdividindo-se os doentes em três grupos segundo a porcentagem de comprometimento linfonodal: ILC-0: 0\%, quando não havia LN comprometidos pela neoplasia; ILC-1: quando existia comprometimento neoplásico em até $20 \%$ do número total de $\mathrm{LN}$ ressecados; ILC-2: quando se encontrou mais de $21 \%$ dos LN ressecados comprometidos pela neoplasia. Tal estratificação foi obtida a partir de cut-off de $20 \%$, baseado no percentil 70, da população selecionada para o estudo (Figura 1 A e B).

Empregou-se estatística descritiva para descrever as características clínicas da casuística selecionada e os dados histopatológicos relacionados à

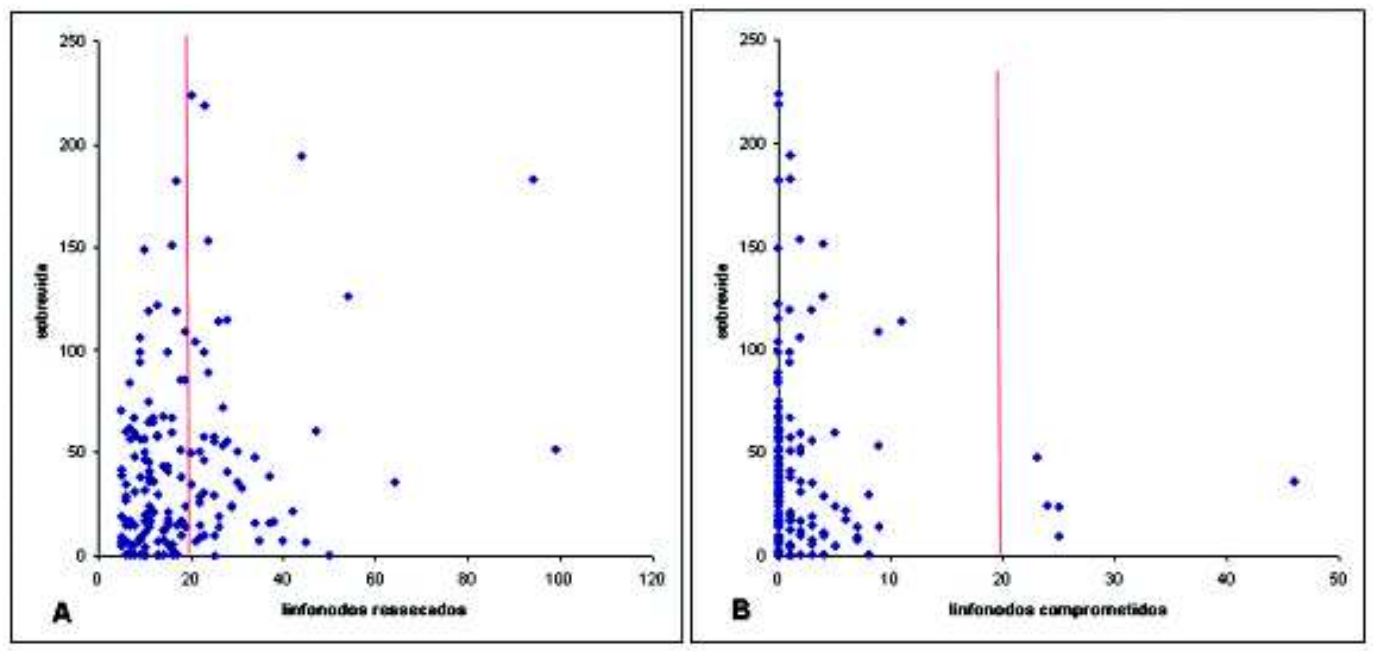

Figura 1-A - Distribuição da amostra elegível segundo o número de linfonodos ressecados. B - Distribuição da amostra elegível segundo Figura 1-A-Distribuição da amostra elegivel segundo o número de
o número de linfonodos comprometidos. Linha de corte em $20 \%$. 
neoplasia. As relações entre o número de LN ressecados, número de LN comprometidos pela neoplasia e o ILC foram determinadas pelo coeficiente de Spearman. A data do óbito em meses foi definida por consulta ao atestado de óbito do doente menos a data da cirurgia A sobrevida em cinco anos foi determinada pelo método de Kaplan-Meyer. Utilizou-se o teste Log-Rank para comparação das curvas de sobrevida. As análises estatísticas univariada e multivariada foram realizadas, para todas as variáveis selecionadas, pelo modelo de Cox, com objetivo de predizer as variáveis de risco independentes relacionada à amostra elegível. A análise dos resultados obtidos foi executada com programa SPSS $^{31}$ para Windows versão 13.0, adotando-se em todos os testes nível de significância de cinco por cento $(\mathrm{p}<0,05)$.

\section{RESULTADOS}

A tabela 1 descreve as características clínicas e anatomopatológicas dos doentes selecionados.

Foram ressecados $2.573 \mathrm{LN}$ e a média dos LN ressecados foi de 22,77 (mínimo de 12 e máximo de 99). Em toda casuística haviam $300 \mathrm{LN}$ comprometidos (mínimo de um e máximo de 46). O tempo médio de seguimento foi de 47,77 $\pm 4,52$ meses.

Sessenta e três doentes $(55,8 \%)$ foram classificados no grupo ILC-0 por não apresentarem comprometimento linfonodal, $33(29,2 \%)$ pertenciam ao grupo ILC-1 por apresentarem $20 \%$ ou menos de comprometimento linfonodal, e $17(15 \%)$ ao grupo ILC-2 por apresentarem mais de $21 \%$ de LN comprometidos.

Não houve correlação entre o número total de $\mathrm{LN}$ ressecados e o ILC $(\mathrm{p}=0,774)$, mas houve correlação entre o número de LN comprometidos e o ILC ( $\mathrm{p}=0,00001$, com rs=0,977). Encontrou-se diferença significante $(p=0,009)$ na sobrevida de cinco anos nos doentes classificados nos diferentes grupos de ILC propostos (Figura 2). Doentes classificados no grupo ILC-0 apresentaram sobrevida em cinco anos maior que $80 \%$, enquanto os classificados no grupo ILC-1 a sobrevida em cinco anos foi de $60 \%$ e, finalmente, para aqueles doentes onde o índice foi maior que $21 \%$ (ILC-2), a sobrevida em cinco anos foi menor que $40 \%$. A análise multivariada demonstrou que o ILC é fator prognóstico independente $(\mathrm{p}=0,009)$.
Tabela 1 - Características anátomo-clínicas da casuística estudada.

\begin{tabular}{|c|c|c|}
\hline Variáveis & $\mathbf{n}$ & $(\%)$ \\
\hline \multicolumn{3}{|l|}{ Gênero } \\
\hline Masculino & 62 & $(54,9)$ \\
\hline Feminino & 51 & $(45,1)$ \\
\hline \multicolumn{3}{|l|}{ Idade } \\
\hline$=70$ anos & 87 & (77) \\
\hline$>70$ anos & 26 & (23) \\
\hline \multicolumn{3}{|l|}{ Raça } \\
\hline Branca & 97 & $(85,8)$ \\
\hline Negra & 10 & $(8,8)$ \\
\hline Amarela & 6 & $(5,3)$ \\
\hline \multicolumn{3}{|c|}{ Grau histológico (diferenciação) } \\
\hline Bem & 27 & $(23,9)$ \\
\hline Moderadamente & 63 & $(55,8)$ \\
\hline Pouco & 23 & $(20,4)$ \\
\hline \multicolumn{3}{|l|}{ Tipo histológico } \\
\hline Intestinal & 90 & $(79,6)$ \\
\hline Mucinoso & 23 & $(20,4)$ \\
\hline \multicolumn{3}{|c|}{ Localizacão do tumor } \\
\hline Cólon proximal & 32 & $(28,3)$ \\
\hline Cólon distal & 42 & $(37,2)$ \\
\hline Reto alto & 37 & $(32,7)$ \\
\hline Sincrônicos & 2 & $(1,8)$ \\
\hline \multicolumn{3}{|c|}{ Estádio classificacão TNM } \\
\hline I & 15 & $(13,2)$ \\
\hline II & 44 & $(38,9)$ \\
\hline IIa & 38 & $(33,6)$ \\
\hline IIb & 6 & $(5,3)$ \\
\hline III & 42 & $(37,3)$ \\
\hline IIIa & 2 & $(1,8)$ \\
\hline IIIb & 24 & $(21.2)$ \\
\hline IIIc & 16 & $(14,3)$ \\
\hline IV & 12 & $(10,6)$ \\
\hline \multicolumn{3}{|c|}{ Infiltração da parede cólica $(\mathrm{T})$} \\
\hline $\mathrm{T}$ & 5 & $(4,4)$ \\
\hline $\mathrm{T} 2$ & 12 & $(10,6)$ \\
\hline T3 & 64 & $(56,6)$ \\
\hline $\mathrm{T}$ & 32 & $(28,3)$ \\
\hline \multicolumn{3}{|l|}{ Linfonodos $(\mathbf{N})$} \\
\hline $\mathrm{N}_{0}$ & 63 & $(55,8)$ \\
\hline $\mathrm{N}_{1}^{0}$ & 27 & $(23,9)$ \\
\hline $\mathrm{N}_{2}^{1}$ & 23 & $(20,4)$ \\
\hline \multicolumn{3}{|c|}{ Invâsão angiolinfática } \\
\hline Presente & 35 & (31) \\
\hline Ausente & 78 & (69) \\
\hline \multicolumn{3}{|c|}{ Número total de linfonodos ressecados } \\
\hline Média & 22,7 & \\
\hline Mediana & 19 & \\
\hline \multicolumn{3}{|c|}{ Índice de linfonodos comprometidos } \\
\hline ILC-0 & 63 & $(55.8)$ \\
\hline ILC- 1 & 33 & $(29,2)$ \\
\hline ILC-2 & 17 & $(15,0)$ \\
\hline
\end{tabular}




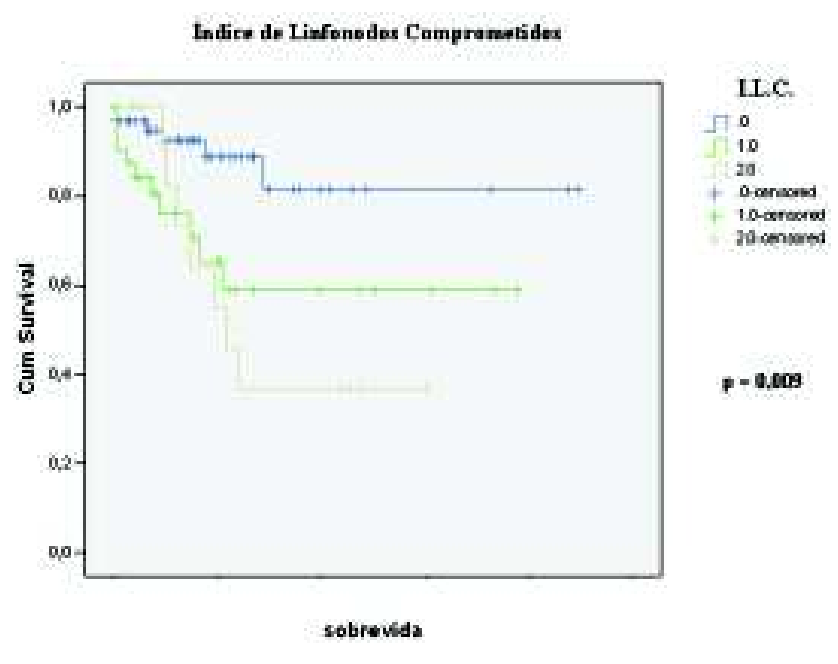

Figura 2 - Sobrevida considerando o índice de linfonodos comprometidos (ILC-0, ILC-1 e ILC-2).

Da mesma forma encontrou-se diferença significante na sobrevida em cinco anos quando comparados doentes com graus comprometimento linfonodal $\mathrm{N}_{0}, \mathrm{~N}_{1}$ e $\mathrm{N}_{2}$ da classificação TNM ( $\left.\mathrm{p}=0,03\right)$ (Figura 3).

Quando se considerou exclusivamente a classificação TNM, verificou-se diferença significante na sobrevida em cinco anos quando os doentes foram subdivididos nos diferentes estádios da classificação ( $\mathrm{p}=$ 0,0001) (Figura 4).

Ao analisar apenas os enfermos pertencentes aos estádios I, II e III, da classificação TNM, excluindo-se, portanto, doentes com metástases (Figura 5) constatou-se que os classificados no grupo ILC-0 apresentaram sobrevida em cinco anos maior que $80 \%$, enquanto os classificados no grupo ILC-1 e ILC-2 a sobrevida em cinco anos foi de $67,6 \%$ e $37,5 \%$ respectivamente $(\mathrm{p}=0,03)$.

\section{DISCUSSÃO}

O CCR é o segundo tipo de câncer mais prevalente em todo o mundo. Estudos demonstram que nos EUA a enfermidade provoca a morte de 23,5 doentes para cada 100.000 habitantes entre os homens, e 16,4 para cada 100.00 habitantes entre as mulheres. ${ }^{(1)}$ Estima-se que o índice relativo de sobrevida global em cinco anos em comparação com a população geral seja de aproximadamente $65 \%$ entre homens e mulheres caucasianas e de aproximadamente $55 \%$ nos indivíduos da raça negra. ${ }^{(1)}$ Entre 2000 e 2004 verificou-se que

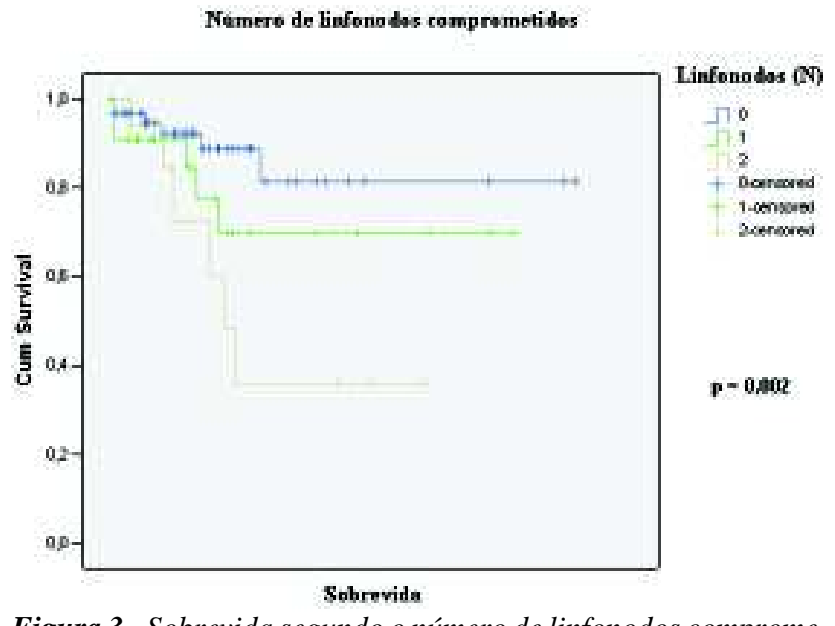

Figura 3 - Sobrevida segundo o número de linfonodos comprome$\operatorname{tidos}\left(N_{0^{\prime}}, N_{l}, N_{2}\right)$.

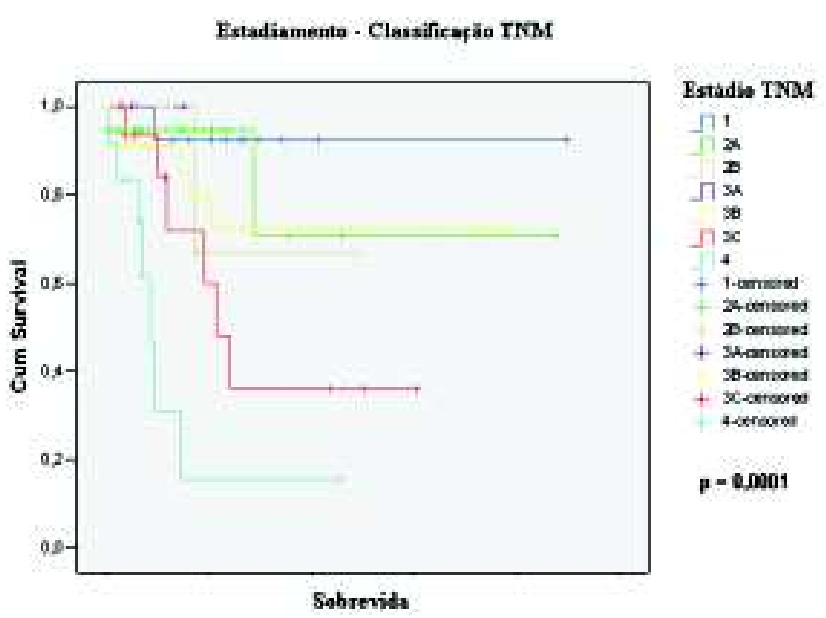

Figura 4 - Sobrevida segundo estádios da classificação TNM.

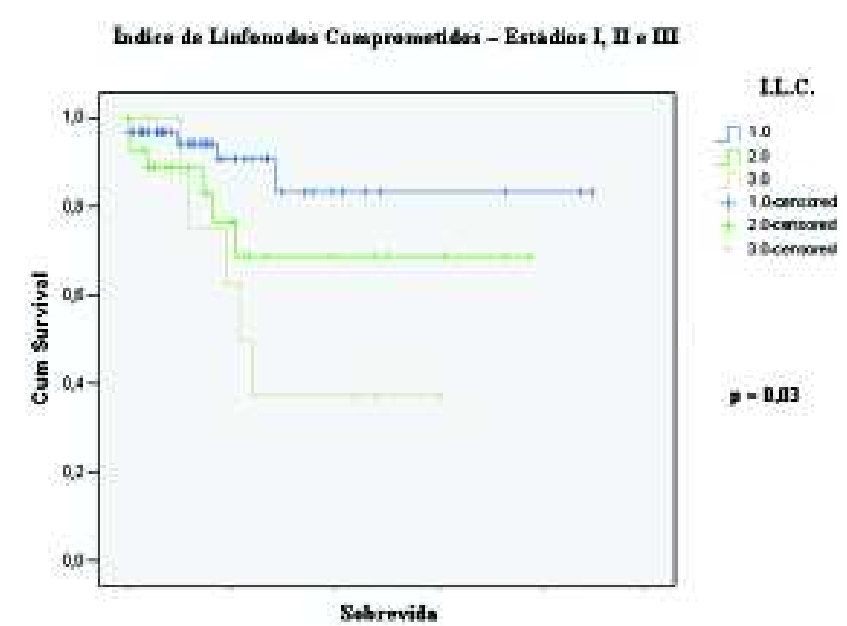

Figura 5 - Sobrevida segundo o índice de linfonodos comprometidos nos doentes classificados nos estádios I, II e III da classificação TNM 
nos EUA ocorreram, em média, 19,4 mortes por CCR em cada 100.000 habitantes. ${ }^{(1)}$

Múltiplos fatores relacionados à sobrevida global e a sobrevida livre de doença nos doentes com CCR têm sido identificados, entretanto, desde os estudos pioneiros de Dukes em 1932(6), a profundidade de invasão do tumor na parede cólica, o comprometimento linfonodal pela doença e a presença de metástases, são os fatores considerados mais relevantes quanto ao prognóstico da enfermidade. Desde então, diferentes propostas de estadiamento para o CCR, incorporaram estes três aspectos principais para sua elaboração. ${ }^{(6,7,7,30,32)}$ Atualmente a proposta de classificação mais utilizada para o estadiamento do CCR preconizada pela AJCC (American Joint Committee on Cancer) e UICC (International Union Against Cancer), baseia-se no sistema TNM (tumor-linfonodometástase), reconhecendo o impacto destes três fatores na sobrevida dos doentes com CCR. ${ }^{(18)}$

Não obstante a importância destas três principais variáveis empregadas nas propostas de estadiamento atualmente utilizadas, outros fatores parecem estar relacionados ao prognóstico da doença. ${ }^{(1-}$ 13) Recentemente, o desenvolvimento de novos métodos de análise da expressão gênica, em particular, por técnicas de tissue microarrays e microarrays, permitiu a observação indireta (imunoistoquímica) ou direta (microarrays) da expressão de proteínas ou de centenas e até mesmo milhares de genes relacionados ao prognóstico da doença, de forma simultânea, tornando possível à identificação do perfil genômico dos diferentes tumores e permitindo a correlação com seu comportamento biológico. ${ }^{(32)}$ Estudos utilizando estas técnicas têm permitido a identificação de um conjunto de genes que funcionam como uma assinatura genética, cuja expressão evidenciou valor prognóstico relacionado à recorrência e sobrevida, superior ao método convencional de estadiamento tumoral como o adotado pela classificação de Dukes e suas variações. ${ }^{(14)}$ Os estudos sugerem que este promissor sistema de estadiamento molecular, pela relação genótipo/fenótipo do tumor, é superior à forma convencional de avaliação prognóstica baseada apenas nos achados morfológicos, como os utilizados pelas classificações de Dukes e TNM. ${ }^{(33)}$ Como primeira aplicabilidade desta inovadora proposta de estadiamento, autores sugerem uma seleção mais acurada para o tratamento quimioterápico, de forma a evitar tratamentos desnecessários ou, até mesmo, sua contra-indicação em ca- sos de possível pior resposta biológica, não detectada pelo estadiamento convencional. ${ }^{(32)}$ Contudo, estas técnicas ainda são dispendiosas e difíceis de serem utilizadas na prática diária, fazendo com que o consagrado sistema de estadiamento baseado na tríade tumorlinfonodo-metástase ainda seja o método de estadiamento, triagem para indicação de terapias adjuvantes e avaliação prognóstica mais utilizado em nossos dias.

Vários estudos têm dado ênfase à importância do estado dos LN como variável determinante do prognóstico do CCR. ${ }^{(18,34,35,36,37)}$ No tratamento definitivo do CCR, a despeito das novas propostas terapêuticas, continua sendo a cirurgia com remoção adequada em monobloco de todo tumor, o tratamento mais eficaz para o CCR, embora ainda existam pontos de vistas contraditórios em relação ao papel desempenhado pela linfadenectomia na sobrevida. ${ }^{(15,17,18)}$ Questiona-se se a linfadenectomia produziria efeito benéfico na sobrevida ou simplesmente seria procedimento que resultaria em estadiamento mais acurado da neoplasia. ${ }^{(15,18)}$ A visão clássica Halstediana acredita que a linfadenectomia é importante para o estadiamento e relaciona-se a sobrevida, enquanto outra vertente aceita que o câncer é doença sistêmica e que a linfadenectomia seria útil apenas para estadiamento mais acurado, não afetando a sobrevida. ${ }^{(17)}$

Objetivando o adequado estadiamento do CCR a importância do número de $\mathrm{LN}$ ressecados tem sido exaustivamente estudada. ${ }^{(15,17,18,33,3,3,35,36,38,39)}$ Estudos analisando a sobrevida do doente em relação ao número de LN extirpados demonstram que a ressecção linfonodal tímida encontra-se relacionada a um pior prognóstico da doença. ${ }^{(15,18)}$ Todavia, o número ideal de LN a serem ressecados ainda apresenta controvérsias variando, segundo a literatura, entre $10 \mathrm{e}$ 17. ${ }^{(17,28,34,35)}$ Nos EUA, o Fórum de Qualidade Nacional e outras organizações definiram, recentemente, que em doentes com CCR um número mínimo de $12 \mathrm{LN}$ devem ser ressecados, sendo este um dos parâmetros utilizados para avaliar a qualidade de um serviço especializado no tratamento da doença. ${ }^{(40)}$ Assim sendo, uma ressecção que contenha menos de $12 \mathrm{LN}$ pode ser inadequada fazendo com que o estado linfonodal não possa ser estabelecido com segurança e melhor classificado como $\mathrm{N}_{\mathrm{x}}$. Baseados nesta recomendação foram selecionados para o presente estudo apenas os doentes que apresentavam 12 ou mais LN extirpados, o que asseguraria maior fidedignidade na avaliação da 
importância do ILC como variável independente de prognóstico quando comparada a outras variáveis sabidamente relacionadas à sobrevida. Apesar de terem sido incluídos doentes com câncer de reto alto, onde o número de $\mathrm{LN}$ extirpados geralmente é menor quando comparados aos tumores de cólon, também foram selecionados apenas doentes que apresentassem mais $12 \mathrm{LN}$ analisados no espécime cirúrgico, o que se encontra em concordância com as melhores recomendações internacionais.

A sobrevida em cinco anos assim como o número de LN ressecados no espécime cirúrgico encontram-se diretamente relacionados com a experiência do cirurgião e ao volume anual de doentes submetidos ao tratamento cirúrgico do CCR em determinado serviço. ${ }^{(41,42)}$ No presente estudo, todos os doentes foram operados por uma mesma equipe treinada para $o$ tratamento cirúrgico do CCR, em Hospital Universitário que é considerado Centro de Referência Regional para o tratamento cirúrgico do CCR onde, em média, são operados, anualmente, aproximadamente 80 casos de CCR e, particularmente, o câncer do reto. É possível que o número de doentes selecionados para a elaboração do presente estudo, que apresentavam ressecções linfonodais dentro dos padrões internacionais, esteja relacionada a estes dois aspectos.

Da mesma forma, a identificação de todos os LN extirpados no espécime cirúrgico também depende da experiência do patologista e da técnica empregada na sua recuperação. ${ }^{(18,25,28)}$ No presente estudo todos os espécimes foram estudados por patologista com experiência em neoplasias do trato digestivo. Com relação à qualidade do espécime ressecado, a cirurgia com remoção adequada em monobloco do cólon contendo margem proximal e distal livres da doença associada à remoção completa do mesocólon relacionado ao tipo de ressecção empreendida, ou do tecido adiposo perirretal íntegro nos tumores de reto, pode influenciar no estadiamento adequado, uma vez que permite que maior número de $\mathrm{LN}$ ressecados possa ser identificado e examinado. Tal fato tem importância ao se considerar que o exame anatomopatológico com número insuficiente de LN ressecados pode levar ao subestadiamento e, conseqüentemente, a não indicação de tratamento adjuvante. Objetivando melhor acurácia no estadiamento, o espécime cirúrgico contendo o mesocólon ou o tecido adiposo perirretal extirpados em monobloco deve ser cuidadosamente manuseado e acondicionado para que o patologista possa identificar o maior número possível de LN. A manipulação inadequada e a violação da integridade do espécime extirpado por pessoas não habilitadas podem dificultar o estudo histopatológico preciso.

Técnicas especiais têm sido preconizadas para possibilitar a identificação de LN com proporções diminutas, por meio da dissolução da gordura com agentes lipolíticos. ${ }^{\left({ }^{3,37)}\right.}$ Haboubi et al. ${ }^{(43)}$, em 1992, avaliando 47 doentes (41 com CCR e seis com doenças não malignas), onde utilizaram a técnica do clareamento da gordura com álcool e xileno, verificaram que empregando técnica convencional de dissecação recuperavam, em média, 6,7 LN por doente, enquanto com a técnica de clareamento passaram a recuperar, em média, 58,2 LN. Cabe lembrar que apesar de seu benefício na detecção de LN menores que $5 \mathrm{~mm}$, que também contribui para um maior número de $\mathrm{LN}$ examinados, existem controvérsias se o tamanho do LN possa ser considerado variável relacionada ao prognóstico da

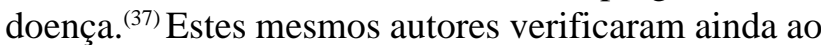
se utilizar técnicas imunoistoquímicas para pesquisar em LN células neoplásicas não identificadas pela técnica da HE houve mudança no estadiamento da classificação de Dukes em 12 dos 41 doentes com CCR, fazendo com que passassem a recomendar a técnica de clareamento e a pesquisa de metástases ocultas por método imunoistoquímico como estratégia válida para evitar o subestadiamento. ${ }^{(42)}$ Entretanto, na prática diária, estas técnicas são laboriosas e dispendiosas, não sendo empregadas ainda de forma rotineira. ${ }^{(36,37,38)}$ No presente estudo estas técnicas de clareamento não foram empregadas, mas mesmo assim pode-se recuperar em média $22,7 \mathrm{LN}$.

Outro potencial problema é identificar o comprometimento do LN pela neoplasia. Os menores índices de sobrevida em significativo grupo de doentes que inicialmente não apresentava comprometimento linfonodal, fez com que autores passassem a analisar o envolvimento linfonodal destes doentes com técnicas mais sofisticadas para identificação de eventuais micrometástases ocultas. ${ }^{(44,45)}$ Verificou-se, com o emprego de técnicas imunoistoquímicas, a possibilidade de ocorrerem mudanças no estadiamento pela identificação de micrometástases em doentes que não apresentavam comprometimento linfonodal quando inicialmente avaliados pela técnica da HE. ${ }^{(42,43)}$ Demonstrouse que 15 a $30 \%$ dos enfermos inicialmente considerados livres de comprometimento linfonodal $\left(\mathrm{N}_{0}\right)$ apresentavam micrometástases quando avaliados pelo es- 
tudo imunoistoquímico. ${ }^{(42,43)}$ Estas evidências sugerem que, caso as micrometástases representem realmente comprometimento do LN por células neoplásicas viáveis, estes doentes tenham sido sub-estadiados.

Embora não exista consenso, estudos demonstraram que, para evitar o subestadiamento, a avaliação do maior número de $\mathrm{LN}$ aumenta proporcionalmente a possibilidade de identificar-se LN comprometidos. ${ }^{(26,34,35)}$ Portanto, este seria um dos fatores prognósticos importantes quando se considera a possibilidade do fenômeno de migração de estádio. ${ }^{(17,26,39)} \mathrm{O}$ conceito de migração de estágio foi primeiramente descrito por Feinstein et al. em 1985, ${ }^{(46)}$ sendo conhecido como fenômeno de Will Rogers. Na verdade trata-se de fenômeno freqüentemente observado na clínica, quando a predição de sobrevida favorável é surpreendida por uma evolução desfavorável. ${ }^{(44)}$ Para evitar o fenômeno de migração de estádio no CCR, a AJCC tem sugerido que a divisão entre pacientes $\mathrm{N}_{1}$ e $\mathrm{N}_{2}$ seja baseada na presença de mais de três LN comprometidos.

De maneira análoga ao comprometimento linfonodal, a relação entre o número de LN comprometidos pela neoplasia e o número de $\mathrm{LN}$ examinados, ILC, vem sendo reconhecido como uma nova variável independente que pode fornecer informações importantes quanto à sobrevida em doentes com câncer. Inicialmente este parâmetro foi utilizado no carcinoma gástrico aonde, atualmente, vem sendo considerado variável prognóstica independente e demonstrando melhor significado e superioridade quando comparada à classificação da AJCC com respeito ao estádio da doença. Estas constatações basearam-se no fato de que a sobrevida dos doentes não era exclusivamente influenciada pela presença de LN comprometidos, mas também pelo número de LN comprometidos. ${ }^{(47)}$ Corroborando estes achados, Bando et al. em 1989, ${ }^{(48)}$ demonstraram no câncer gástrico, decréscimo de $50 \%$ no fenômeno da migração de estádio ao se utilizar sistema que considerava o ILC. Dessa maneira, o uso do ILC para o estadiamento do câncer gástrico diminuiu a incidência deste fenômeno quando comparado à classificação proposta pela AJCC. Desde então, uma série de artigos vem sendo publicados demonstrando a relação entre o ILC e a sobrevida de doentes com câncer gástrico. ${ }^{(19,20,45,46)}$

A partir destas evidências Berger et al. ${ }^{(9)}$ evidenciaram, pela primeira vez, resultados semelhantes para o CCR quando verificaram por análise multivariada, em casuística significativa e formada por grupos uniformes de doentes, que o ILC foi considerado variável prognóstica significante nos enfermos que apresentavam de 10 a 15 ou mais que 15 LN ressecados, porém não para doentes com menos de $10 \mathrm{LN}$ ressecados no espécime cirúrgico. ${ }^{(25)}$ Outros estudos confirmaram a importância do ILC como variável relacionada a sobrevida no CCR. ${ }^{(24,26,27,29,49)}$ Recentemente, Wang et al., em 2008, ${ }^{(26)}$ avaliando o ILC em grande número de doentes (24.477 casos), selecionando apenas casos de câncer do cólon no estádio III da classificação TNM, referem que os doentes classificados nos estádios IIIB e IIIC se comportam como um grupo heterogêneo, sendo a maioria deles sobrestadiados ou sub-estadiados. Os autores confirmaram que o ILC foi a variável prognóstica de maior acurácia para o correto estadiamento de doentes com câncer do cólon estádio III. Os resultados do presente estudo parecem ir de encontro a tais achados. Ao se analisar, isoladamente, doentes portadores de tumores classificados nos estádios I, II e III da classificação TNM utilizando-se o ILC, verificou-se que doentes classificados como ILC-1 apresentavam sobrevida significativamente maior que os classificados como ILC-2 $(\mathrm{p}=0,03)$.

No presente estudo os doentes foram estratificados segundo o ILC em três grupos, divididos segundo a proporção entre LN comprometidos e analisados ser de $0 \%$, até $20 \%$ e maior que $21 \%$. Ao se adotar cut off de $20 \%$ para a estratificação dos grupos, pequena população com LN comprometidos estava situada acima da linha de corte, não se observando o mesmo quando se considerou o número de LN examinados. O seguimento de doentes com CCR que apresentam LN comprometidos demonstra sobrevida em cinco anos menor que $70 \%$. Dessa forma, a exemplo da amostra analisada pelo presente estudo, torna-se adequado para estratificar os doentes nos três grupos de ILC propostos, adotar percentil 70, o que na presente casuística correspondeu a uma linha de corte de $20 \%$. Caso fosse reduzida a linha de corte, estar-se-ia colocando maior parcela da população estudada com LN comprometidos além desta linha, o que geraria maior significância dos dados estatísticos, ao se comparar a proporção de comprometimento relacionada à sobrevida, além de se estar desobedecendo à história biológica da evolução destes tumores.

Somente um único estudo não pode considerar

o ILC como variável independente de prognóstico no 
câncer do cólon na análise multivariada. Todavia, na análise univariada, considerando valor de significância menor ou igual a $10 \%$, a relação entre prognóstico do câncer de cólon e o quociente de LN acometidos/dissecados (ILC) foi significante. Talvez estes achados estejam relacionados ao fato que mais da metade dos doentes $(58,1 \%)$ selecionados no estudo pertenciam ao estádio IV da classificação TNM - doentes sabidamente com pior prognóstico - e que o número de $\mathrm{LN}$ considerados para análise multivariada foi de 10 e mesmo assim em pequeno número de doentes. ${ }^{(28)}$ Os próprios autores justificam que as possíveis explicações para o fato do ILC não predizer a sobrevida de forma independente foram a heterogeneidade da amostra estudada em relação ao estadiamento inicial do tumor, número de $\mathrm{LN}$ dissecados no ato cirúrgico, curto tempo de seguimento dos doentes, pequena casuística comparada a outros estudos e a não avaliação de fatores que poderiam estar relacionados a sobrevida, tais como presença de co-morbidades graves, e complicações pósoperatórias. $^{(28)}$

Não obstante ao avaliar doentes com câncer de reto alto, onde o número de $\mathrm{LN}$ ressecados geralmente é menor que no câncer de cólon o presente estudo pode demonstrar que o ILC revelou-se uma variável independente e significante na determinação da sobrevida de doentes com CCR $(\mathrm{p}=0,009)$. Os pacientes classificados como LNR-0 apresentaram sobrevida global em cinco anos maior que $80 \%$, enquanto em pacientes com LNR-1 a sobrevida foi de cerca de $60 \%$, e para aqueles classificados como LNR-2 foi menor que $40 \%$ em cinco anos.

Apesar do papel da linfadenectomia ainda ser controverso na presente casuística o envolvimento linfonodal mostrou-se como fator prognóstico importante, quando se observou sobrevida de cinco anos em mais de $75 \%$ dos pacientes com envolvimento exclusivo da parede do cólon sem comprometimento linfonodal $\left(\mathrm{N}_{0}\right)$, em contraste com a proporção de $60 \%$ e $30 \%$ para aqueles que apresentam comprometimento dos $\mathrm{LN} \mathrm{N}_{1}$ e $\mathrm{N}_{2}$, respectivamente. Corroboram este fato a correlação significante entre número de LN comprometido e o ILC. De modo semelhante ao já observado, não foi possível encontrar relação entre o número de $\mathrm{LN}$ ressecados e o número de $\mathrm{LN}$ comprometidos representados pelo ILC. ${ }^{(50)}$

O ILC não deve ser encarado como um substituto para a adequada ressecção linfonodal no CCR. ${ }^{(25)} \mathrm{O}$ número ideal de $15 \mathrm{LN}$, ou pelo menos 12
LN, deve ser obtido para a adequada avaliação do envolvimento linfonodal, bem como do próprio ILC. ${ }^{(15,16,17,18,25)}$ Demonstrou-se no presente estudo que nos doentes com mais que $12 \mathrm{LN}$ ressecados, independentemente do tumor situar-se no cólon ou reto, que o ILC foi fator prognóstico independente para a sobrevida em cinco anos. Estes achados demonstram que, mesmo em pacientes que tem sua avaliação limitada a $12 \mathrm{LN}$ examinados, o ILC tem um dramático impacto no correto estadiamento e sobrevida do CCR. Desta forma, a avaliação de doentes com CCR baseada no ILC é um potencial fator para informações quanto à estratificação e prognóstico, podendo auxiliar na melhor adequação da terapia adjuvante. Doentes com menos de $12 \mathrm{LN}$ examinados foram excluídos do presente estudo por apresentarem ressecção linfonodal aquém do número ideal preconizado pela literatura atual. Contudo, ao analisarmos o impacto do ILC neste grupo de pacientes, que não foram considerados no presente estudo, e adotando-se os mesmos níveis de corte para a estratificação proposta, verificamos que o ILC também estava correlacionado com a sobrevida em cinco anos ( $\mathrm{p}=0,003$, dados não apresentados).

Como conclusão, o ILC se mostrou uma importante variável prognóstica em doentes com CCR. ${ }^{(25)}$ Estudos multicêntricos e com casuística expressiva vêm mostrando a importância do número de $\mathrm{LN}$ positivos, número de LN examinados, e do ILC em doentes com CCR. ${ }^{(25,26)} \mathrm{O}$ ILC pode decrescer o fenômeno de migração de estádio que pode ocorrer quando o patologista examina um número insuficiente de LN, quer por ressecção linfonodal tímida, quer por técnica patológica inadequada. Estudos avaliando a presença de micrometástases nos LN ressecados, e o seu impacto na relação com o ILC, poderão acrescentar novas evidências que minimizem, ainda mais, o fenômeno de Will Rogers.

Atualmente, estamos estudando, na mesma casuística, com ênfase especial aos doentes que não apresentavam LN comprometidos quando avaliados pela técnica convencional da HE (estádios I e II), mas que tiveram pior sobrevida, a presença de micrometástases nos LN extirpados. O objetivo deste novo estudo é verificar, utilizando técnica imunoistoquímica com anticorpos primários anticitoqueratinas (AE-1 e AE-3), a presença de LN comprometidos por micrometástases em doentes inicialmente considerados livres de comprometimento linfonodal 
pela técnica convencional. É possível caso esta possibilidade se confirme, que uma nova estratificação do ILC, considerando que a presença de micrometástases melhore, ainda mais, a acurácia do índice na predição da sobrevida destes enfermos.

\section{CONCLUSÃO}

Os resultados do presente estudo permitem concluir que o ILC é um método facilmente reprodutível, que apresenta acurácia prognóstica, podendo ser considerado uma variável independente preditora de sobrevida em doentes com CCR.

\section{AGRADECIMENTO}

A Profa. Dra. Marcia Pivatto Serra responsável pela Disciplina de Estatística da Universidade São Francisco, Bragança Paulista, pela análise estatística adotada no presente estudo.

ABSTRACT: In patients with colorectal cancer, the compromising of the lymph node is one of the most important prognostic factors. Aim: The aim of this study is to determine the prognostic independent value of relation between compromised and examined lymph nodes, (LNR) in patients with colorectal cancer. Method: One hundred and thirteen 113 patients (62 women) with colon ad upper rectum cancer were studied. Fifteen patients were staged to the stadium I of the TNM classification, 44 to II, 42 to III and 24 to the stadium IV. The lymph node ratio rate was determined by the relation between total number of compromised and examined lymph nodes. The patients were divided in three groups according to the proportion of compromised lymph nodes: LNR-0: when there was no lymph node involvement; LNR-1: when there was compromising of up to $20 \%$ of the examined lymph nodes and LNR-2: when there was compromising in $21 \%$ or more of the examined nodes. The relations between lymph node ratio, number of removed lymph nodes and number of compromising lymph nodes by cancer were determined. Patients younger than 18 years old who were submitted to neoadjuvant quimioradiation protocol were excluded as well as the ones who had less than 12 lymph nodes removed. The clinical and histopathological variables were analyzed through descriptive statistic. The correlation between the variables was valued by Spearman correlation test. The five years survival was determined by the tests of KaplanMeier, Log-rank and the multivariate analysis of the prognostic variables by the Cox model establishing level of significance of $5 \%(\mathbf{p = 0 . 0 5})$. Results: There was significant difference in the five years overall survival in patients classified in the different groups of LNR ( $\mathrm{p}=0.009$ ). Patients of the group LNR-0 presented five years overall survival bigger than $80 \%$, while in those classified to the groups LNR-1 and LNR-2, the five years overall survival was less than $60 \%$ and $40 \%$, respectively. The number of compromising lymph nodes alone was correlated to the five years overall survival $(\mathrm{p}=\mathbf{0 . 0 3}$ ). The multivariate analysis demonstrated that the LNR is an independent prognosis variable of the five years overall survival $(\mathrm{p}=0.009)$. There was relation between the total number of compromised lymph nodes and the LNR ( $p=0.00001$, with $r s=0.977)$. Conclusion: The results of the present study showed that the LNR can be considered an independent prognostic variable of overall survival in patients with colorectal cancer.

Key Words: Lymph Nodes; Lymph Node Excision; Prognosis; Colorectal Neoplasms; Survival Analysis; Survival Rate.

\section{REFERÊNCIAS}

1. Surveillance epidemiology and end results (SEER). National Cancer Institute. [cited 2008 mar 21]. Avaliable from: http:// seer.cancer.gov/statfacts/html/colorect/html

2. Sanfelippo PM, Beahrs OH. Factors in the prognosis of adenocarcinoma of the colon and rectum. Arch Surg.1972;104:401-6.

3. Cain AS, Longino LA. Carcinoma of the colon in children. J Pediatr Surg. 1970; 5:527-32.

4. Wolmark N, Wieand HS, Rockette HE, Fischer B, Glass A, Lawrence $\mathrm{W}$, et al. The prognostic significance of tumor location and bowel obstruction in Dukes B and C colorectal cancer. Findings from the NSABP clinical trials.1983;198:74352 .
5. Grinnel RS. The grading and prognosis of carcinoma of the colon and rectum. Ann Surg.1939;109:500-33.

6. Dukes CE. The classification of cancer of the rectum. J Pathol.1932;35:323-32.

7. Astler VB, Coller FA. The prognostic significance of direct extension of carcinoma of the colon and rectum. Ann Surg.1954;139:846-51.

8. Dukes CE, Bussey HJR. The spread of rectal cancer and its effect on prognosis. Br J Cancer.1958;12:309-20.

9. Halvorsen TB, Sein E. Influence of mucinous components on survival in colorectal adenocarcinomas: a multivariate analysis. J Clin Pathol.1988;41:1068-72.

10. Martinez CAR, Priolli DG, Cardinalli IA, Piovesam H, Pereira JA, Waisberg J, et al. Correlação entre o padrão de distribuição tecidual e os valores séricos do antígeno carcinoembrionário em doentes com câncer. Rev bras Coloproct.2006; 26:41-53. 
11. Cutait R. Contribuição ao estudo do antígeno carcinoembrionário e dos antígenos de superfície ABH no câncer colo-retal. Tese (Livre Docência) - Faculdade de Medicina da Universidade de São Paulo, São Paulo, 1991. 96p.

12. Khankhanian N, Mavligit GM, Russell WO, Schimek M. Prognostic significance of vascular invasion in colorectal cancer of Dukes' B class. Cancer.1977;39:1195-200.

13. Cruz JV, Cutait R, Martinez CAR, Bevilacqua RG, Leite KRM, Margarido NF. Importância prognostica da invasão neural no câncer colorretal. Estudo imunoistoquímico com a proteína S100. Rev bras Coloproct.2006;26:280-93

14. Wang Y, Jatkoe T, Zhang Y, Mutch MG, Talantov D, Jiang J, et al. Gene expression profiles and molecular markers to predict recurrence of Dukes' B colon cancer. J Clin Oncol.2004;221:564-71.

15. Sigurdson ER: Lymph node dissection: Is it diagnostic or therapeutic? J Clin Oncol 2003;21:965-7.

16. Gajra A, Newman N, Gamble GP, Kohman LJ, Graziano SL. Effect of number of lymph nodes sampled on outcome in patients with stage I non-small-cell lung cancer. J Clin Oncol.2003; 21:1029-34.

17. Wong JH, Severino R, Honnebier MB. Number of nodes examined and staging accuracy in colorectal carcinoma. J Clin Oncol.1999;17:2896-900.

18. LeVoyer TE, Sigurdson ER, Hanlon AR, Mayer RJ, MacDonald JS, Catalano PJ. Colon cancer survival is associated with increasing number of lymph nodes analyzed: a secondary survey of intergroup trial INT-0089. J Clin Oncol.2003; 21:2912-9.

19. Celen O, Yildirim E, Berberoglu U. Prognostic impact of positive lymph node ratio in gastric carcinoma. J Surg Oncol.2007;96:95-101.

20. Persiani R, Rausei S, Biondi A, Boccia S, Cananzi F, D’Ugo D. Ratio of metastatic lymph nodes: Impact on staging and survival of gastric cancer. Eur J Surg Oncol.2007;34:519-24.

21. Herr HW, Bochner BH, Dalbagni G, Donat SM, Reuter VE, Bajorin DF. Impact of the number of lymph nodes retrieved on outcome in patients with muscle invasive bladder cancer. J Urol. 2002;167:1295-8.

22. Weir L, Speers C, D'yachkova Y, Olivotto IA. Prognostic significance of the number of axillary lymph nodes removed in patients with node negative breast cancer. J Clin Oncol.2002;20:1793-9.

23. Berger AC, Watson JC, Ross EA, Hoffman JP. The metastatic examined lymph node ratio is an important prognostic factor after pancreaticoduodenectomy for pancreatic adenocarcinoma Am Surg.2004;70:235-40.

24. Lee HY, Choi HJ, Park KJ, Shin JS, Kwon HC, Roh MS, et al. Prognostic significance of metastatic lymph node ratio in node-positive colon carcinoma. Ann Surg Oncol.2007; 14:1712-7.

25. Berger AC, Sigurdson ER, Le Voyer T, Hanlon A, Mayer RJ, Macdonald JS, et al. Colon cancer survival is associated with decreasing ratio of metastatic to examined lymph nodes. J Clin Oncol.2005;23:8706-12

26. Wang J, Hasset JM, Dayton MT, Kulaylat MN. Lymphy node ratio: role in the staging of node-positive colon cancer. Ann Surg Oncol. 2008;15:1600-8.

27. Derwinger K, Carlsson G, Gustavsson B. A study of lymph node ratio as a prognostic marker in colon cancer. Eur J Surg Oncol. 2007;34:771-5.

28. Trufelli DC, da Costa Miranda V, Palos CC, Ramos E, Abrão MN, da Silva VA, et al. Positive /total dissected lymph nodes ratio as a prognostic factor in colorectal cancer. Rev Ass Med Bras.2007;53:539-42.

29. Schumacher P, Dineen S, Barnett C Jr, Fleming J, Anthony T The metastatic lymph node ratio predicts survival in colorectal cancer. Am J Surg.2007;194:827-31.

30. Nelson H, Petrelli N, Carlin A, Couture J, Fleshman J, Guillem J, et al. National Cancer Institute Expert Panel. Guideline 2000 for Colon and Rectal Cancer Surgery. J Nat Cancer Inst.2001;93:583-96.

31. SPSS $^{\oplus}$ Inc.; Ilinois, USA

32. Collaborative staging and coding manual. American Joint Committee on Cancer. [cited 2008 mar 21]. Avaliable in URL from: http://www.cancerstaging.org

33. Pinho MSL. Estadiamento molecular do câncer colorretal: o futuro se aproxima. Rev bras Coloproct.2005;25:279-84.

34. Hermanek P, Giedl J, Dworak O. Two programs for examination of regional lymph nodes in colorectal carcinoma with regard to the new pN classification. Path Res Pract.1989;185:867-73.

35. Goldstein NS, Sanford W, Coffey M, Layfield LJ. Lymph node recovery from colorectal resection specimens removed for adenocarcinoma: Trends over time and a recommendation for a minimum number of lymph nodes to be recovered. Am J Clin Pathol.1996;106:209-16.

36. Chen SL, Bilchik AJ. More extensive nodal dissection improves survival for stages I to III of colon cancer: a population-based study. Ann Surg.2006;244:602-10.

37. Herrera-Ornelas L, Justiniano J, Castillo N, Petrelli NJ, Stulc JP, Mittelman A. Metastases in small nodes from colon cancer. Arch Surg.1987;122:1253-6.

38. Mönig SP, Baldus SE, Zirbes TK, Schröder W, Lindemann DG, Dienes HP, et al. Lymph node size and metastatic infiltration in colon cancer. Ann Surg Oncol.1999;6:579-81.

39. Wood TF, Saha S, Morton DL, Tsioulias GJ, Rangel D, Hutchinson $\mathrm{W}$ Jr, et al. Validation of lymphatic mapping in colorectal cancer: In vivo, ex vivo, and laparoscopic techniques. Ann Surg Oncol.2001;8:150-7.

40. Wong SL, Ji H, Hollenbeck BR, Morris AM, Baser O, Birkmeyer JD. Hospital lymph node examination rates and survival after resection for colon cancer. JAMA.2007;298:2149-54.

41. Schrag D, Panageas KS, Riedel E, Hsieh L, Bach PB, Guillem $\mathrm{JB}$, et al. Surgeon volume compared to hospital volume as a predictor of outcome following primary colon cancer resection. J Surg Oncol.2003;83:68-78. 
42. Meyerhardt JA, Castelano PJ, Schrag D, Ayanian JZ, Haller DG, Mayer RJ, et al. Association of hospital procedure volume and outcomes in patients with colon cancer at high risk for recurrence. Ann Inter Med. 2003;139:649-57.

43. Haboubi NY, Clark P, Kaftan SM, Schofield PF. The importance of combining xylene clearance and immunohistochemistry in the accurate staging of colorectal carcinoma. J R Soc Med.1992;85:386-8

44. Palma RT, Waisberg J, Bromberg S, Simão AB, Godoy AC Micrometastasis in regional lymph nodes of extirpated colorectal carcinoma: immunohistochemical study using anti-cytokeratin antibodies AE1/AE3. Colorectal Dis. 2003:5 $164-8$

45. Messerini L, Cianchi F, Cortesini C, Comin CE. Incidence and prognostic significance of occult tumor cells in lymph nodes from patients with stage IIA colorectal carcinoma. Hum Pathol. 2006:37:1259-67.

46. Feinstein AR, Sosin DM, Wells CK: The Will Rogers phenomenon: Stage migration and new diagnostic techniques as a source of misleading statistics for survival in cancer. $\mathrm{N}$ Engl J Med.1985;312:1604-8.
47. Inoue K, Nakane Y, Iiyama H, Sato M, Kambara T, Nakai K, et al. The superiority of ratio-based lymph node staging in gastric carcinoma. Ann Surg Oncol.2002;9:27-34.

48. Bando E, Yonemura Y, Taniguchi K, Fushida S, Fujimura T, Nishimura G, et al. Outcome of ratio of lymph node metastasis in gastric carcinoma. Ann Surg Oncol.2002;9:775-84

49. Mammen JM, James LE, Molloy M, Williams A, Wray CJ, Sussman JJ. The relationship of lymph node dissection and colon cancer survival in the Veterans Affairs Central Cancer Registry. Am J Surg.2007:194;349-54.

50. Bui L, Rempel E, Reeson D, Simunovic M. Lymph node counts, rates of positive lymph nodes, and patient survival for colon cancer surgery in Ontario, Canada: a populationbased study. J. Surg Oncol.2006;93:439-45.

Endereço para correspondência: DENISE GONÇALVES PRIOLLI

Rua São Vicente, 614

Atibaia - São Paulo - CEP: 12947-390

$\mathrm{Tel} / \mathrm{Fax}$. (11) 4412-7730

e-mail: depriolli@ @erra.com.br 